DISCUSSION NOTE

\title{
The Principle of Causal Completeness: Reply to Daniel von Wachter
}

\author{
Michael Esfeld*
}

This ${ }^{1}$ discussion note is motivated by the fact that, to my mind, Daniel von Wachter does not represent the position of the friends of the principle of the causal closure of the physical world (PCC) in a completely fair manner in his paper "The principle of the causal openness of the physical" in this issue. What Kim (1998, chap. 2) and Papineau (2002, chap. 1) among others defend is this principle:

PCC: For any physical event $p$, insofar $p$ has a cause, it has a complete physical cause.

PCC is therefore better be called "principle of causal completeness" rather than "closure." PCC is a claim about what holds in the actual universe. Thus, it is a contingent principle. Possible worlds in which PCC is false are not ruled out. PCC is not committed to determinism, since it leaves open to what extent physical events are caused. It only states that if there are causes of physical events, there are complete physical causes.

PCC can be employed in an argument for physicalism in the sense of the metaphysical stance according to which everything that exists - or at least everything that causes something - is physical. To turn PCC into an

1 This is a reply to (von Wachter 2019).

* University of Lausanne

Department of Philosophy, University of Lausanne, CH-1015 Lausanne, Switzerland

$\triangle$ Michael-Andreas.Esfeld@unil.ch Website: http://www.michaelesfeld.com/

(C) The Author. Journal compilation (C) The Editorial Board, Organon F. 
argument for physicalism, one has to endorse a further principle that excludes systematic overdetermination: if physical events have causes, these causes do not systematically overdetermine these events. Hence, if (by PCC) physical events have physical causes insofar as they have causes at all, there are no additional non-physical causes that systematically cause these events as well. This then yields the exclusion of non-physical causes for physical events. In contrast to PCC, the usual argument to rule out systematic overdetermination is indeed an a priori argument against banning causes that do not make a difference. Be that as it may, systematic overdetermination plays no role in the case for the causal openness of the physical that von Wachter seeks to make. It would be of no help for the metaphysical stance according to which non-physical minds (souls) or God can cause physical events: if they do so, they do not overdetermine these events. If they intervene in the physical domain, $\mathrm{PCC}$ is false, also in the non-modal version given above.

What, then, is the argument for PCC? The argument is that if the physical laws are true, PCC is true as well. In other words, the argument is that scientific realism with respect to physical laws is committed to PCC. For this argument, it is irrelevant what the physical laws are - that is, whether they are the laws of classical mechanics, of the standard model of elementary particles in quantum field theory, of a future theory of quantum gravity, etc. Furthermore, it is irrelevant whether they are deterministic or probabilistic. The argument is that taking the laws of our physical theories (classical, relativistic, quantum) as guide to the true laws of nature, anyone who is a scientific realist is committed to PCC. Again, scientific realism is not at disposal in von Wachter's paper. The issue hence is whether this argument is correct. In other words, von Wachter has to show us a way how to be a scientific realist and, yet, reject PCC, also in its non-modal version as stated above.

I have reservations about the manner in which von Wachter analyses physical theories with respect to making a case for separating scientific realism from the commitment to PCC. Consider Newtonian physics. According to Newton, every change in the state of motion of a physical object is due to forces influencing the motion of the object. It is true that Newton's second law leaves open what these forces are. Hence, there could in principle also be forces originating in a non-physical agent that influence the motion 
of physical objects - provided that these forces respect also the third law, that is, the principle of action and reaction corresponding to each other, so that there also would have to be a reaction on the non-physical agent. The problem with that, obviously, is that a non-physical agent does not have a state of motion. Be that as it may, the central issue is that the three laws of Newton are a framework for the formulation of physical theories. But they are not a physical theory themselves.

The physical theory is gravitation. Von Wachter is right in pointing out that Laplacean determinism follows from Newtonian gravitation only if gravitation is the only force. But this does not open up a way to separate scientific realism from PCC. The point at issue is that Newtonian gravitation sets up the paradigm for a fundamental and universal physical theory, that is, a theory that applies to everything in the universe and that cannot be reduced to another theory. According to Newton's theory of gravity, every physical object interacts with every other physical object in the universe via gravitation (insofar as the objects have a mass). Hence, there is a single law that relates all the objects in the universe with one another. More precisely, the law relates the state of motion of all the objects in the universe at a given time with the state of motion of all the objects in the universe at any other-past or future - time. Formulating the law as a differential equation, on the left hand side figure the positions and velocities of all the objects in the universe and on the right hand side figure the variables that determine the temporal evolution of the positions and velocities in the case of gravitation.

This way of conceiving physical laws applies to modern physics as a whole, whatever the details and differences are in classical, relativistic and quantum physics. Laws are formulated in terms of differential equations such that the variables figuring on the right hand side of the equation indicate what determines the temporal evolution of the objects as they figure on the left hand side of the equation. Over and above gravitation, these are variables for electromagnetism, the weak and the strong interaction. The important issue is that given these variables, the temporal evolution of the objects is fixed. According to our current state of knowledge, the temporal evolution of the physical objects is fixed by these four types of interaction. The fact that we do not have (as yet) one single equation that brings all these four types together is not relevant for present purposes. Furthermore, 
it is not relevant for present purposes whether future physics will find more types of interaction of the same kind as gravitation, electromagnetism, the weak and the strong interaction or reduce them to less types of interaction. What is relevant is that one cannot add to this structure of physical theories formulated in terms of differential equations that indicate what determines the temporal evolution of the objects under consideration a clause to the effect that something may intervene from the outside that stops the evolution of the objects as indicated by what figures on the right hand side of the differential equation in question.

Of course, a law that relates the state of motion of all the objects in the universe at one time to the state of motion of all the objects in the universe at other times is as such of no practical use and cannot even be tested. The law has to contain a procedure how to apply it to specific systems in the universe that we can observe or control while abstracting from the rest of the universe. Furthermore, it has to tell us something about the evolution of specific systems that we can expect under ignorance of their exact initial conditions. That is why even a universal, deterministic law has to be linked with a probability measure that enables the derivation of propositions about the evolution of ensembles of systems that are prepared under identical conditions when ignoring their exact initial conditions (such as, for instance, the distribution of the outcomes in a large series of coin tosses). It is clear how to do this both in classical and quantum physics. In brief, these demands do not call the mentioned structure of physical theories in question.

Nonetheless, like von Wachter, I think that the link between scientific realism and PCC is not as firmly established as these considerations may suggest. However, to my mind, the point at issue is not the mentioned structure of physical theories, but the metaphysics of laws. One should be very cautious about using terms such as forces acting on objects. In physics, when objects interact, there is nothing that travels from one object to other objects and that literally pushes them to move in a certain manner. There is no justification to associate forces with agents that literally act on objects. Recall the following statement of Russell in his famous critique of the notion of cause:

In the motions of mutually gravitating bodies, there is nothing that can be called a cause and nothing that can be called an 
effect; there is merely a formula. Certain differential equations can be found, which hold at every instant for every particle of the system, and which, given the configuration and velocities at one instant, or the configurations at two instants, render the configuration at any other earlier or later instant theoretically calculable. [...] This statement holds throughout physics, and not only in the special case of gravitation. (Russell 1912, 14)

Whatever one thinks about Russell's views on causation and his philosophy in general, what he says about the physics here is certainly correct. This is already evident if one considers the formulation of physical determinism: the propositions stating the laws of nature and the propositions describing the state of the world at an arbitrary time (i.e. the propositions describing the initial conditions) entail the propositions describing the state of the world at any other time. Hence, determinism in physics is - onlyabout entailment relations among propositions. There is nothing here that suggests that what there is in the state of the world at a given time literally produces or brings about the evolution towards the future. The question for a scientific realist then is what it is in the world that makes these propositions true, that is, in virtue of what in the ontology these entailment relations among propositions hold.

As an example of a parsimonious ontology of physics that is nevertheless rich enough for these propositions to come out true in virtue of what there is in the world, consider Humeanism about laws of nature. On Humeanism, first comes the motion of the matter in the universe, then come the laws. They are theorems of the best system, that is, the system that strikes the best balance between being simple and being informative in representing the motion of the matter in the universe. Hence, the laws are only fixed on the basis of the motion of the matter throughout the entire history of the universe. What is relevant in the present context is that Humeanism about laws is compatible with the idea that some motion of matter in the universe depends on the free will of minds that may be non-physical entities, and, yet, when it comes to representing the motion of the matter in the universe in the best system, the variables that enter into this system are only ones for universal physical interactions [see my paper in this volume (Esfeld 2019)]. 
On the stance that can be dubbed Super-Humeanism, what the Humean says about laws applies to the dynamical variables that are functionally defined in terms of their role for the particle motion - such as mass, charge, energy, fields, an initial wave function in quantum physics, etc. Their values are not intrinsic to the state of the universe at any given time, but depend on the motion that the matter in the universe actually takes [see (Esfeld and Deckert 2017, chap. 2.3)]. Consequently, again, what these values are depends on the actual motion of matter, and there is the conceptual space open for maintaining that these values are - in part-influenced by motions that humans make out of their (non-physical) free will. To put it in a nutshell, if humans had chosen to do otherwise out of their (non-physical) free will, on Humeanism, the laws of nature would be slightly different [see (Beebee and Mele 2002)]; on Super-Humeanism, the initial values of some of the dynamical variables that enter into the laws of nature would have been slightly different.

In sum, when entering the metaphysics of mind, it seems to me that we should not touch upon the sketched structure of physical theories and scientific realism as regards that structure. The metaphysics of laws of nature is rich enough to leave the conceptual space for a large variety of positions in the metaphysics of the mind open.

\section{References}

Beebee, Helen, and Alfred Mele. 2002. "Humean Compatibilism." Mind 111 (442): 201-23. https://doi.org/10.1093/mind/111.442.201

Esfeld, Michael. 2019. "Why Determinism in Physics Has No Implications for Free Will." Organon F 26 (1): 62-85. https://doi.org/10.31577/orgf.2019.26105 Esfeld, Michael, and Dirk-André Deckert. 2017. A Minimalist Ontology of the Natural World. New York: Routledge.

Kim, Jaegwon. 1998. Mind in a Physical World. An Essay on the Mind-Body Problem and Mental Causation. Cambridge (Massachusetts): MIT Press.

Papineau, David. 2002. Thinking about Consciousness. Oxford: Oxford University Press. https://doi.org/10.1093/0199243824.001.0001

Russell, Bertrand. 1912. "On the Notion of Cause." Proceedings of the Aristotelian Society 13: 1-26.

von Wachter, Daniel. 2019. "The Principle of the Causal Openness of the Physical." Organon F 26 (1): 40-61. https://doi.org/10.31577/orgf.2019.26104 